\title{
Parental knowledge and attitudes to oral health
}

\author{
The relationship between socio-demographic characteristics and dental health knowledge and attitudes of parents \\ with young children N. J. Williams, J. G. Whittle and A. C. Gatrell Br Dent J 2002; 193: 651-654
}

\section{Objective}

To determine if parental socio-demographic characteristics are associated with dental knowledge and attitude.

\section{Design}

A questionnaire survey of 500 consecutive parents with children aged approximately 8-months, attending clinics in 1999/2000, in Burnley, Pendle and Rossendale, for health visitor distractionhearing tests.

\section{Outcome measures}

Scores were obtained for dental knowledge and attitudes. The socio-demographic variables of parental ethnicity, age, education and area of residence were used to determine any associations.

\section{Results}

Parental age ranged between 16-46 years. Child age ranged between 7-11 months. Significant differences were detected for parental dental knowledge according to ethnicity $(p=0.003)$, educational status $(p=0.000)$, and area of residence $(p=0.016)$. Significant differences were also found in dental attitudes; ethnicity $(p=0.000)$, educational status $(p=0.004)$ and area of residence $(p=0.005)$. Parental age was not significant for either knowledge or attitude.

\section{Conclusions}

Lack of further education, being Asian and living in a deprived area means parents have less chances of high dental knowledge and positive dental attitudes.

\section{IN BRIEF}

- This study has found strong relationships between parental sociodemographic characteristics and levels of dental knowledge and attitude.

- Lack of further education, living in a deprived area and being Asian meant that parents had less chance of scoring highly for levels of dental knowledge and having a positive dental attitude.

- Multiple logistic regression analysis revealed that the ethnicity of parents was the factor of most significance above deprivation.

- This knowledge may allow oral health promotion interventions to be targeted at those communities who have the greatest need.

\section{COMMENT}

Parental oral health knowledge and attitudes undoubtedly have a role in determining the oral health of young children. This study has explored the knowledge and attitudes of parents of infants attending for a health visitor check. The results highlight the impact of socio-demographic factors in this sample from the North West of England. Deprivation, ethnicity and educational status all emerged as significant factors influencing parent's oral health knowledge and attitudes. What are the implications of these findings for promoting the oral health of pre-school children and reducing inequalities?

It is now generally agreed that implementing interventions that merely aim to improve the population's oral health knowledge and attitudes will have a limited long term effect on oral health. Indeed dental health education may increase inequalities within the population. Instead, what is required is the adoption of a broader approach which aims to tackle the range of factors which determine health and oral health. 'Joined up' interventions which focus on working in partnership with relevant agencies to create long term sustainable improvements in health are needed. To be successful these interventions need to work with the local communities to develop appropriate solutions.

As the authors of this paper state, Sure Start Initiatives provide an opportunity for oral health professionals to become involved in collaborative programmes aimed at reducing health inequalities. It is essential however that Sure Start programmes base their work upon a contemporary evidence base. In many parts of the country oral health promoters are engaged in a variety of innovative health programmes working with Sure Start staff. Evaluating interventions and disseminating examples of good practice is very important to avoid duplication of effort.

Dr Richard G. Watt, Reader and Specialist in Dental Public Health, University College London. 